\title{
Top-Down TDMA Scheduling Algorithm in Wireless Sensor Networks
}

\author{
Ho-Ting Wu, Chih-Ching Chen, Kai-Wei Ke, and Song-Ferng Wang
}

\begin{abstract}
This paper aims at the design of a centralized-controlled TDMA scheduling algorithm in the single channel Wireless Sensor Network (WSN) environments. We consider the network scenario where packets generated by each WSN device are destined for the common Gateway. A Top-down scheduling mechanism is proposed to assign wireless devices near the Gateway with high priority for transmitting packets. Through simulations, this paper compares and analyzes the scheduling efficiency and packet delay performance achieved by the proposed mechanism and other related works under various network topologies and scenarios. We also investigate the capability of these algorithms in providing differentiated service to those packets with multiple frequency generation patterns and different priority orders. Furthermore, the lossy wireless channel with $P D R<1$ is also considered to evaluate the reliability performances. Performance results have revealed that the proposed mechanism can achieve satisfactory results in terms of packet delay and packet loss probability for lots of network scenarios.
\end{abstract}

Index Terms-Wireless sensor network, time division multiple access, centralized scheduling algorithm, top-down.

\section{INTRODUCTION}

With the advance of wireless sensing technique, we have witnessed the widespread applications of wireless sensor networks (WSN) in recent years. Wireless sensor networks [1], [2] often consist of one or more gateways as well as lots of sensing devices. A gateway, which serves as the data link, collects sensed data sent from sensing devices and forwards them to a backbone server for further processing. The sensing devices, equipped with various sensing modules, generate packets with sensed data from time to time. All of these packets are destined for the gateway. For certain network topologies, sensing devices also have to serve as the relays/routers to forward packets towards the gateway.

In the single channel WSN environment, all WSN devices share the same wireless channel for data transmission. Therefore, without the deployment of a proper medium access control (MAC) mechanism, more than one device may transmit packets simultaneously. Such transmission often leads to data collision and loss of data, resulting in waste of time and energy. The CSMA/CA (Carrier Sensing Multiple Access/Collision Avoidance) algorithm is commonly employed in wireless networks. However, it may induce the

Manuscript received October 20, 2015; revised January 22, 2016. This research is supported by Ministry of Science and Technology of Taiwan, R.O.C. project number: MOST-104-2221-E-027-055.

Ho-Ting Wu, Chih-Ching Chen, Kai-Wei Ke, and Song-Ferng Wang are with the Department of Computer Science and Information Engineering, National Taipei University of Technology, Taipei, 106, Taiwan (e-mail: htwu@csie.ntut.edu.tw). well-known hidden node problem [3], causing the unpredictable packet collision and then packet retransmission. Therefore, to tackle such problem and meet the performance requirement imposed by certain critical WSN network environments, the TDMA (Time Division Multiple Access) algorithm, which allocates packet transmission in a deterministic manner, can be employed to ensure no packet collision and guaranteed performances, if properly designed. One key issue for the TDMA employment is the design of the scheduling mechanism to determine which nodes to transmit/receive packet at any time epoch. Both centralized and distributed TDMA schemes [4] are possible with distinguished pros and cons, respectively. In this paper, we focus on the design of the centralized TDMA scheduling algorithm, where a central controller arbitrates and schedules transmission for all network devices.

The remainder of this paper is organized as follows. Backgrounds and related works are presented in Section II, followed by descriptions of our proposed algorithms in Section III. The performance results and comparisons are conducted in Section IV. Conclusions are drawn in Section V.

\section{BACKGROUNDS AND RELATED WORKS}

We consider a wireless network scenario with one gateway and a few sensing devices. Each sensing device will generate packets to report its sensed data towards the gateway either periodically or non-periodically. The single gateway serves as the data link. Therefore, the convergecast traffic pattern where all the packets generated by sensing devices are destined for the common gateway is considered. Since the distance between a faraway sensing device and the gateway may be far beyond one hop distance, some wireless devices may have to serve as the intermediate relay nodes or routers to forward such packets toward the gateway. For such network application, the graph of a tree topology rooted at the gateway can be constructed through the execution of a routing algorithm. Thus in this paper, a tree architecture rooted at the gateway is assumed to be readily constructed via some sort of routing path selection criterion, which is beyond the scope of this paper. We consider the upward traffic only, where every child device transmits self-generated or forwarded packets to its single parent device.

The TDMA MAC access scheme is assumed for the operation. The time axis is divided into multiple slots, where a single packet can be transmitted and acknowledged within one slot duration. Every sensing device maintains the same global clock in the synchronized manner. After executing a scheduling algorithm, a central scheduler generates a scheduling table, indicating which nodes to transmit/receive 
packets at any particular time slot. Such scheduling table is broadcast to all sensing devices before the system operation. Therefore, any single device resides in either one of the following three - transmission, reception, or idle - states at any time instance according to the given scheduled table. In this way, the system efficiency and performance highly rely on the design of the centralized TDMA scheduling algorithm for a given tree topology and traffic load.

Due to the low cost consideration of the WSN implementation, the half-duplex transmission mode is assumed. That is, any single device cannot transmit or receive packets simultaneously. In addition, to avoid possible packet collision which may be due to the hidden node problem in the single channel TDMA wireless environment, devices within 2-hop distances cannot transmit packets at the same time slot. Otherwise, an intended receiver of a particular transmission may also receive packets destined for other receivers at the same time due to the inherent broadcast feature of wireless transmission. On the other hand, those devices located more than 2-hop away may be allocated to transmit their packets at the same time slot without collision by taking the advantage of spatial reuse property. Therefore, an efficient TDMA scheduling algorithm is to find those devices which can transmit packets simultaneously without packet collision at each time slot and then construct the scheduling table accordingly to satisfy the performance requirements, such as packet delay or packet dropping rate etc.

Such non-conflicting nodal pair finding problem can be mapped into the distance-2 graph coloring problem [5] in the graph, where nodes with the same color in the graph denote those devices which can transmit packets at the same slot. It can be shown to be a NP-complete problem when trying to find a minimum length of slot duration to schedule all packets to reach the gateway for a given load. Therefore, a heuristic scheduling algorithm is usually resorted to for solving this problem.

The authors in [6] proposed a Time-Optimal scheduling algorithm for such convergecast traffic problem. Every node alternated among one of the following three states: $\mathrm{T}$ (ransmission), I(dle) and R(eception) during one cycle of three time slots. The algorithm was first applied to the linear topology and was then generated to a tree topology. In [7], the authors proposed two centralized scheduling algorithms: the Node-Based algorithm and the Level-Based algorithm.

For the Node-Based algorithm [7], all nodes in the graph were first ordered in the non-increasing order of their associated degrees. Then all non-conflicting nodal pairs were constructed and assigned one particular color for each nodal pair in sequence, where all nodes of the same nodal pair were assigned the same color. The scheduling process then took place after the coloring processing finished. At each slot of this process, one color was selected. All nodes assigned the same color were allowed to transmit packets at that particular slot. Color selection was carried out in a round robin fashion in general. However, at the beginning of each slot, if none of all nodes corresponding to the particular color had any packets to transmit, the color was skipped and the next color in sequence was then picked. In this way, it was guaranteed that there was at least one packet transmission at each slot. No single idle slot was allocated without packet transmission.
The scheduling process continued until all packets in the network were delivered to the gateway.

There were three steps to implement the Level-Based algorithm [7]. (1) Step 1: The linear network construction: The original tree network was first compressed into a linear network with the same depth, where a node of such linear network represented all nodes at the same level of the original tree network. (2) Step 2: Coloring the linear network: Any coloring algorithm could be implemented in such network. (3) Step 3: Scheduling the original network: the process was similar to the scheduling process of the Node-Based algorithm. Nodes at different levels but with the same color could send packets using the same slot. In addition, nodes at the same level could also send packets simultaneously as long as they were 2 hops away in the original tree network.

\section{Proposed TOP-DOWN SCHEDULING ALGORITHM}

We propose a TDMA scheduling algorithm, the Top-down approach, for the single channel WSN application, aiming at minimizing packet latency. In the Top-down scheduling mechanism, devices near the Gateway are assigned with higher priorities to transmit packets, in general. The coloring and scheduling process are combined and carried out at each scheduling slot. At the start of each slot, a node nearest to the gateway is selected among those nodes with buffered packets for transmission. If at least two candidate nodes are available, the node with the most number of packets in the subtree rooted at this node is selected. If there is still more than one candidate available, a node is randomly selected among these nodes. Such node is called the pivot node. Starting from the pivot node, a coloring process is performed downward to find all nodes which can transmit packets together with the pivot node simultaneously without causing packet collision. Therefore, all these nodes are able to transmit packets at this slot. Such scheduling and coloring process is repeated at every slot until all packets in the network were delivered to the gateway.

Take the tree topology of Fig. 1 as an example to illustrate how the Top-down algorithm carries out the scheduling and coloring process. The two nodes connected by the dash line (---) link are within one hop wireless transmission range even though this link is not used as the routing path of the topology. However, it should be as a link for the distance- 2 coloring problem. Assume that each node in the tree has one packet waited for transmission initially. At the beginning of the first scheduling slot, those nodes at the first level- nodes 1, 2, 3are selected for comparisons. Since node 1 has the largest number of packets in the subtree rooted at itself compared with node 2 and node 3 , node 1 is thus selected as the pivot node of this slot. Then, starting from node 1 , the coloring process is performed. Both node 6 and node 7 at level 2 satisfy the distance- 2 coloring criteria and thus are included as the same non-conflicting nodal pair as node 1 . Continue the coloring process for nodes at level 3 at this slot and cannot find any nodes to satisfy the criteria. It is noted that node 8 is still within the 2 hop distance with node 6 , since there exists a dash line between node 5 and node 6 . Therefore, nodes 1,6 and 7 are scheduled for packet transmission at slot 1 . For slot 
2 , node 1 has no buffered packet for transmission. Node 6 is then selected as the pivot node, since it has more packets in the subtree rooted at itself than node 7 does. Node 5 is found to be at the same nodal pair with node 2 for this slot. Repeat the same pivot node selection and coloring process for remaining slots until all packets reach the gateway; the final scheduling table is then constructed as in Table I. It shows that 9 slots are required to complete the packet transmission for such topology.

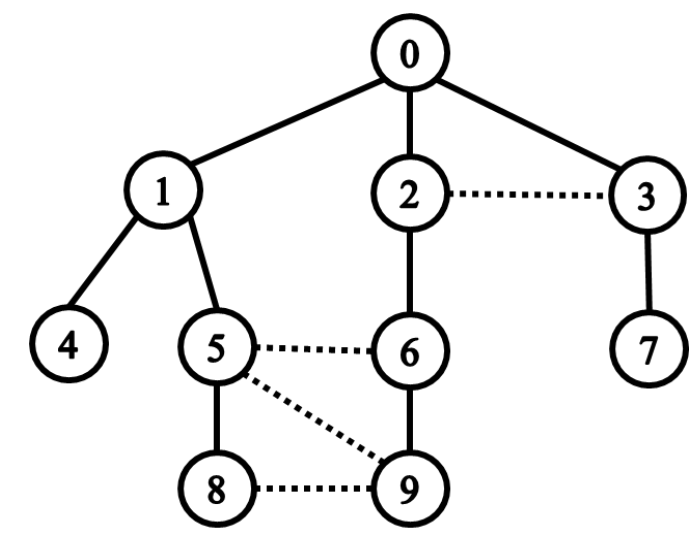

Fig. 1. A tree topology.

TABLE I: THE SCHEDULING TABLE
\begin{tabular}{|c|c|c|c|c|c|c|c|c|c|}
\hline \#slot & 1 & 2 & 3 & 4 & 5 & 6 & 7 & 8 & 9 \\
\hline \multirow{3}{*}{ \#ID } & 1 & 2 & 1 & 2 & 1 & 3 & 1 & 2 & 3 \\
\cline { 2 - 11 } & 6 & 5 & 9 & 4 & 6 & 5 & & & \\
\cline { 2 - 10 } & 7 & & & 8 & & & & & \\
\hline
\end{tabular}

The aforementioned top-down scheduling algorithm is applied well to the single priority traffic in WSN applications. However, sensing devices may generate multiple frequency sensed data for various scenarios [8]. Data with higher frequency usually have stricter delay requirement than those with lower frequency. Such data are thus considered to own a higher priority. In addition, non periodical data or bursty data such as alarming message contains the highest priority. Therefore, the top-down scheduling algorithm is extended as follows to provide differentiated service (DS) for sensed data with various priorities.

At each slot, nodes with the highest priority packets are first considered for scheduling and coloring process from those located nearest the gateway downward, as the above mentioned top down scheduling mechanism. After those possible candidate nodes with the highest priority packets are selected, nodes with the second highest priority packets are then considered for the same slot, following the similar top down approach. The procedure is repeated until nodes with each priority packets are considered. This completes the nodal pair process for this slot. Similarly, the same approach continues for subsequent slots until all packets reach the gateway.

\section{Simulation RESUlts AND COMPARISONS}

The OMNET++ simulation platform is used to carry out the simulations. We compare the performance results for the network employing either Time-Optimal, Node-Based, Level-Based or the proposed Top-Down scheduling algorithm. Convergecast traffic pattern is assumed for all the carried out simulations.

For the first simulation scenario, we consider the network topology to be linear, star or tree topology. The total slots and the average packet delay are evaluated as the number of sensing devices changes from 1 to 30 . The total slots denote the total number of slots required to allow for all packets reaching the gateway if each node has only one packet for transmission initially. The transmission priority is the same for every packet. We assume that the wireless channel quality is excellent such that the PDR (packet delivery rate) $=1$ for each link. All the delay performance is measured in terms of slot time, where the duration of a single slot is equal to $10 \mathrm{~ms}$. One slot can accommodate the transmission of a single packet and its associated acknowledged packet.

The average packet delay performances for linear topology are revealed in Fig. 2. The Top-down scheduling algorithm achieves the shortest average packet delay performance since by employing this algorithm, packets near the gateway are given relatively better advantages to reach the gateway as soon as possible. The Node-based algorithm results in the highest average packet delays among all mechanisms. Since the results of total slots for this case are about the same for all algorithms employed, we omit such figure for the brevity of the paper.

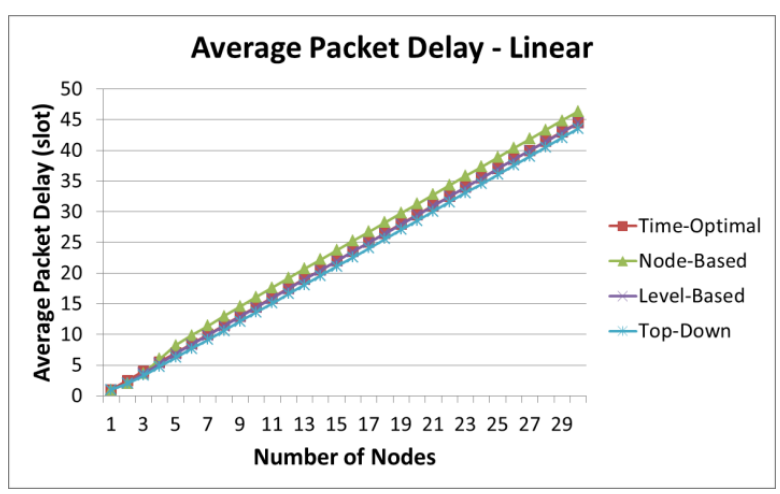

Fig. 2. The average packet delay for linear topology.

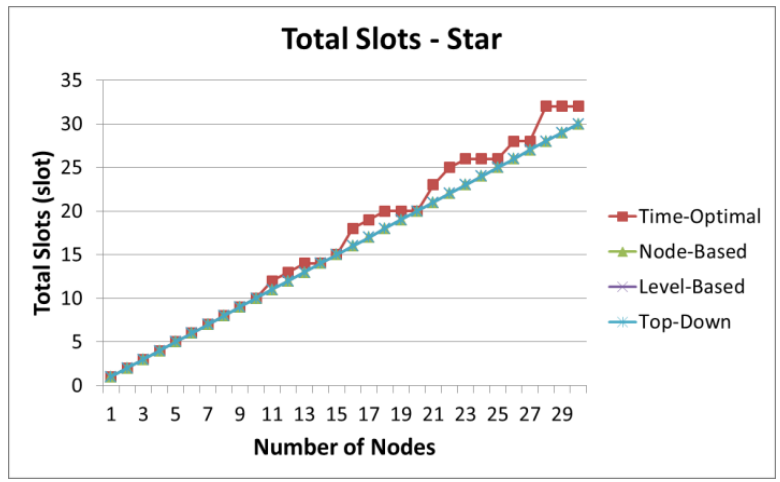

Fig. 3. The total slots for star topology.

The performance results for the star topology are shown in Fig. 3. It is shown that the Time-Optimal scheduling algorithm exhibits the worst performance for the total slots performance, since every node has to follow the three state transition rule, which causes a few idle slots without any packet transmission. The other three algorithms yield the same performance for such simple case because exactly one packet is sent to the gateway at each slot by a single sensing 
device due to the half-duplex limitation of the gateway. A similar result for the average packet delay performance of such star topology is observed among these four mechanisms. Thus, we also omit the figure. A more general network topology, tree topology, is then considered. Fig. 4 and Fig. 5 reveal the performances for the average packet delay and total slots performances, respectively. The proposed Top-down algorithm is shown to achieve the best results for both performance indexes, followed by the Node-Based algorithm. The Time-Optimal mechanism results in the worst performance.

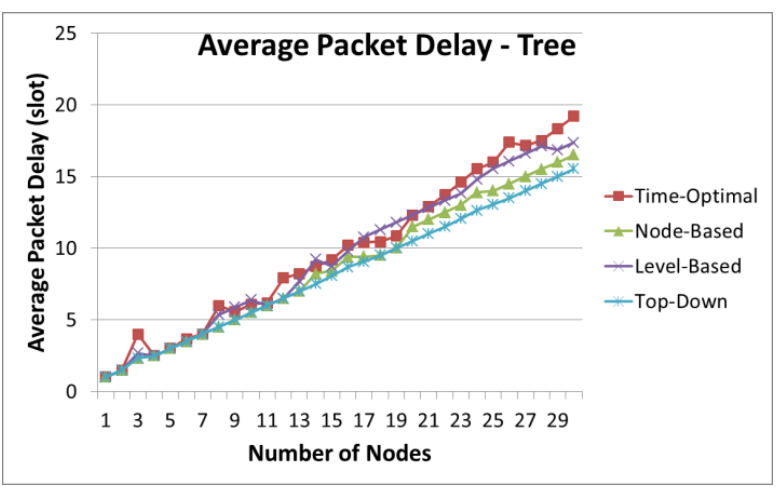

Fig. 4. The average packet delay for tree topology.

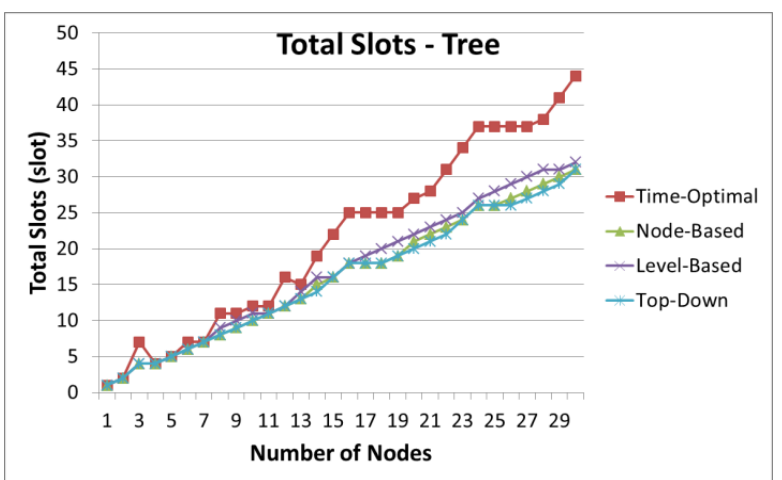

Fig. 5. The total slots for tree topology.

For the second simulation scenario, we limit our focus on a tree network with 10 sensing devices as shown in Fig. 6. Each of these sensing devices generates non-periodical data, P1, as well as two different kinds of periodical traffics, P2 and P3. The P1 traffic is generated in a Poisson pattern with mean inter-arrival time of 10 seconds. The P2 and P3 traffics carry the regular sensed data and are generated periodically with the periods of 1 and 5 seconds, respectively. We assume that P1 traffic carries the most important message such as the alarming data in the case of emergency. Therefore, the priority order is set as $\mathrm{P} 1>\mathrm{P} 2>\mathrm{P} 3$. That is, a sensing device always transmits $\mathrm{P} 1$ packets first, followed by $\mathrm{P} 2$ and $\mathrm{P} 3$ at its scheduled time slots. Since P1 represents the highest priority traffic, no packet dropping policy is imposed for such traffic. However, the periodical P2 traffic will be dropped if it is not able to reach the gateway within 1 second. So does P3, if it cannot reach the gateway within 5 seconds. We assume the lossy channel condition with PDR varied from 0.2 to 1 for each link. That is, the link error probability of each link is 1-PDR, randomly for every slot. A loss packet is retransmitted by the sensing device of the underlying link. Since the periodical data are predictable, the central scheduler thus constructs the scheduling table according to these traffic patterns only. The total simulation time is set as 1 hour. We collect the simulation results of average packet delay experienced by each traffic type and the packet loss rate of $\mathrm{P} 2$ and P3 data for various scheduling algorithms employed.

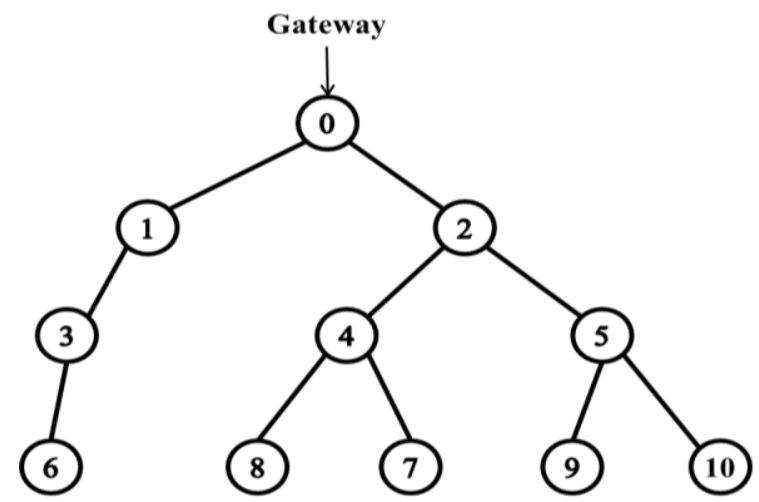

Fig. 6. The simulated tree topology.

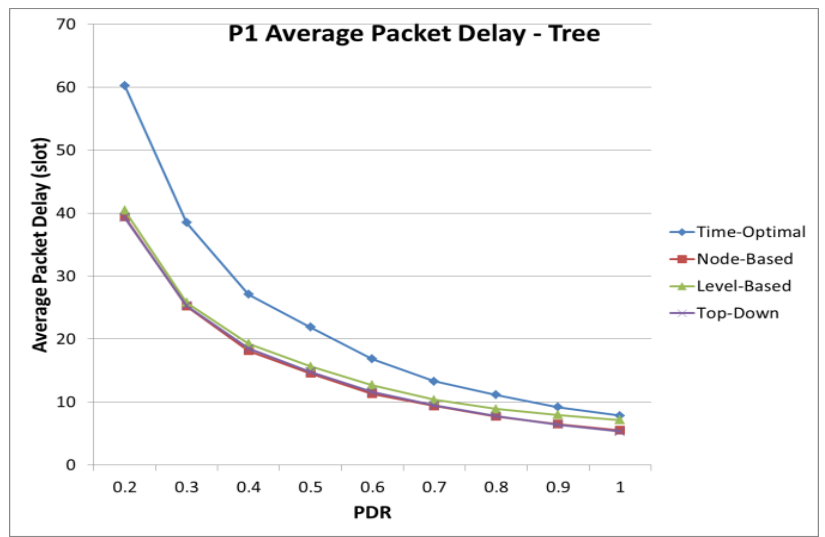

Fig. 7. The average packet delay of P1 traffic for tree topology.

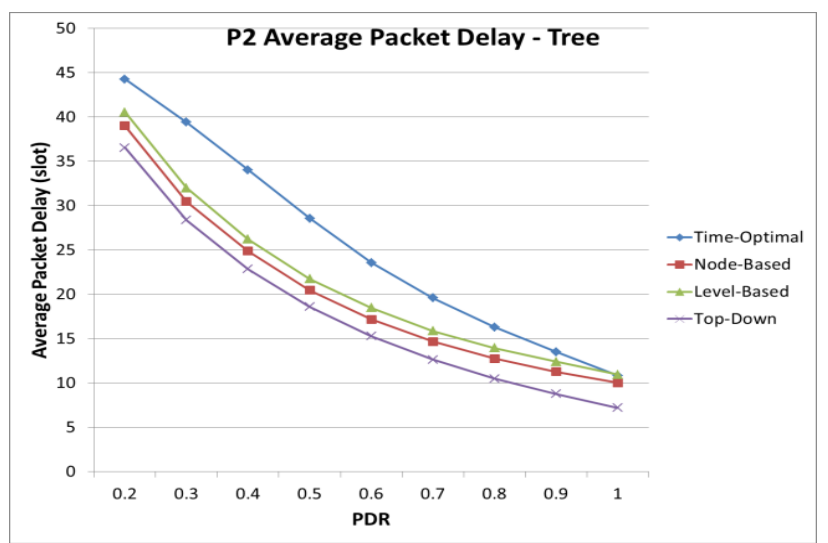

Fig. 8. The average packet delay of P2 traffic for tree topology.

Fig. 7 reveals the average packet delay of P1 traffic. In general, as the PDR increases, the number of retransmission decreases, leading to lower average packet delay as expected. It is observed that Top-down approach yields the shortest packet delay, followed closely by the Node-Based algorithm while the Time-Optimal method generates the longest average packet delay. Fig. 8 and Fig. 9 show the performances of P2 traffic and Fig. 10 and Fig. 11 exhibit those of P3 traffic. We still observe the similar performance behaviors among the four scheduling mechanisms. The Top-down algorithm generates the lowest packet delay and packet loss rate for P2 and $\mathrm{P} 3$ traffics. 


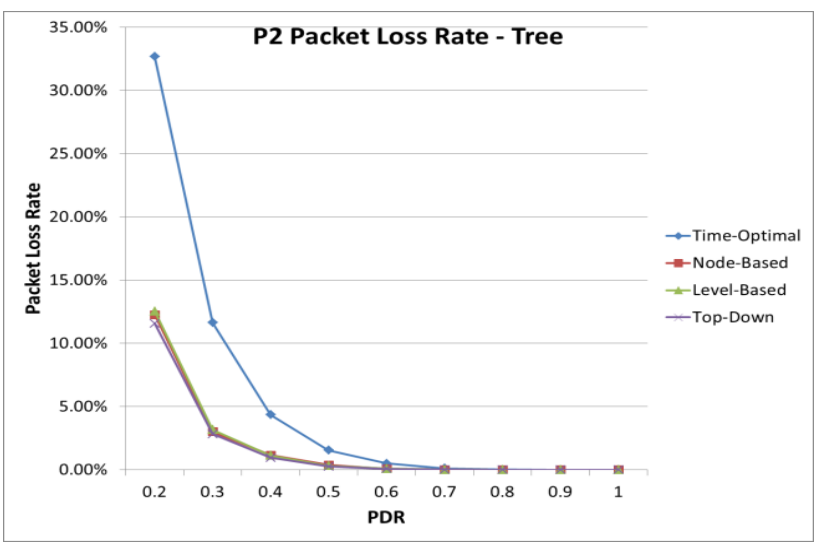

Fig. 9. The packet loss rate of P2 traffic for tree topology.

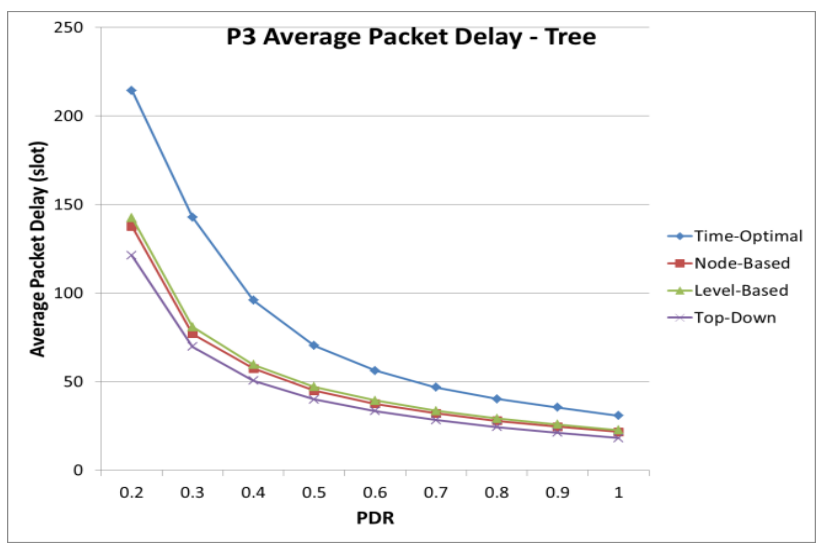

Fig. 10. The average packet delay of P3 traffic for tree topology.

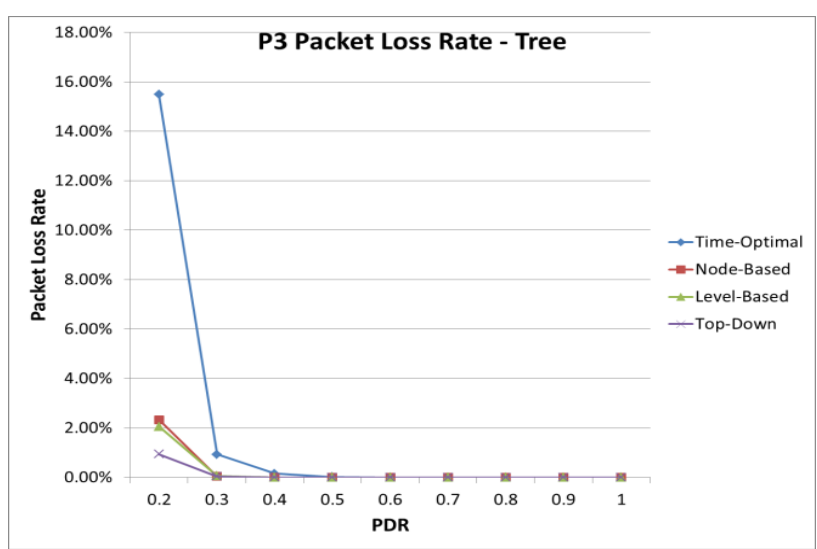

Fig. 11. The packet loss rate of P3 traffic for tree topology.

\section{CONCLUSIONS}

The Centralized Top-down TDMA scheduling algorithm is proposed in this paper for employment in the WSN scenarios. This mechanism allows nodes near to the gateway with high priority for packet transmission, leading to the low packet delay for the convergecast traffic pattern. Simulations are conducted and performance results are compared with those from related scheduling methods. The Top-down approach is shown to achieve satisfactory performance results in terms of packet delay and packet loss rate for various networking scenarios.

\section{REFERENCES}

[1] A. A. K. Somappa, K. Ovsthus, and L. M. Kristensen, “An industrial perspective on wireless sensor networks - A survey of requirements, protocols, and challenges," IEEE Communications Surveys and Tutorials, vol. 16, no. 3, pp. 1391-1411, 2014.

[2] H. Zhang, P. Soldati, and M. Johansson, "Performance bounds and latency-optimal scheduling for convergecast in wireless HART networks," IEEE Trans. on Wireless Commun., vol. 12, no. 6, pp. 2688-2696, Jun. 2013.

[3] J. Kurose and K. Ross, Computer Networking: A Top-down Approach, 6th ed. Pearson, U.S.A., 2013.

[4] P. Huang, Li Xiao, S. Soltani, M. Mutka, and N. Xi, "The evolution of MAC protocols in wireless sensor networks: A survey," IEEE Communications Surveys \& Tutorials, vol. 15, no. 1, pp. 101-120, 2013.

[5] S. McCormick, "Optimal approximation of sparse hessians and its equivalence to a graph coloring problem," Math. Programming, vol. 26, no. 2, pp. 153-171, 1983.

[6] S. Gandhama, Y. Zhang, and Q.-F. Huang, "Distributed time-optimal scheduling for convergecast in wireless sensor networks," Computer Networks, vol. 52, no. 3, pp. 610-629, Feb. 2008.

[7] S. C. Ergen and P. Varaiya, "TDMA scheduling algorithms for wireless sensor networks," Wireless Networks, vol. 16, no. 4, pp. 985-997, May 2009.

[8] M. A. Yigitel, O. D. Incel, and C. Ersoy, "QoS-aware MAC protocols for wireless sensor networks: A survey," Computer Networks, vol. 55, no. 8, pp. 1982-2004, Feb. 2011.

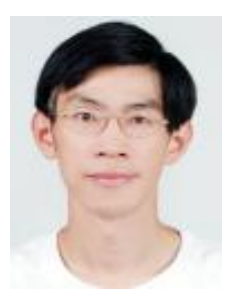

Ho-Ting Wu received his B.S. degree from National Taiwan University in 1986, and both the M.S. and Ph.D. degrees from University of California, Los Angeles, USA, in 1989 and 1994, all in electrical engineering. From 1994 to 1996, he was a lecturer in the Department of Electrical Engineering at Chang Gung University, Taiwan. Since 1996, he has been a faculty of National Taipei University of Technology, Taiwan, where he is currently a professor in the Department of Computer Science and Information Engineering. His current research interests include multimedia communications, optical and wireless networks.

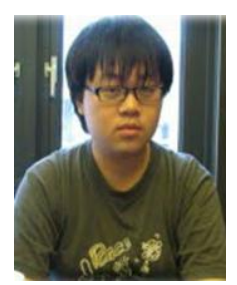

Chih-Ching Chen obtained his M.S. degree from the Department of Computer Science and Information Engineering at National Taipei University of Technology, Taiwan in 2015. He was with the Research Laboratory of Wireless and Broadband Networks, under the supervision of Prof. Ho-Ting Wu. His current research interests include computer networking and computer system design.

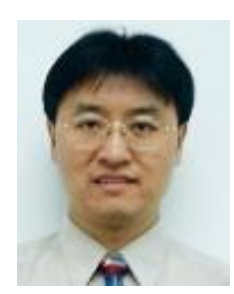

Kai-Wei Ke received the Ph.D. degree in electrical engineering from Georgia Institute of Technology, USA, in 1996. Currently, he is a professor of computer science and information engineering, National Taipe University of Technology, Taiwan, ROC. His research interests include traffic modeling, Internet protocols, and call admission, resource allocation, routing algorithms, and performance evaluation for wireless multimedia communication networks.

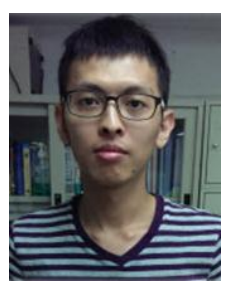

Song-Ferng Wang obtained his M.S. degree from the Department of Computer Science and Information Engineering at National Taipei University of Technology, Taiwan in 2015. He was with the Research Laboratory of Wireless and Broadband Networks, under the supervision of Prof. Ho-Ting Wu He is currently with HTC Inc., working on the design of smartphones. His research interests include the designs of communication network protocol and application layer interface. 